\title{
A NEW CONSTRUCTION FOR CANCELLATIVE FAMILIES OF SETS
}

\author{
James B. Shearer \\ IBM Research Division \\ T.J. Watson Research Center \\ P.O. Box 218 \\ Yorktown Heights, NY 10598 \\ email: jbs@watson.ibm.com
}

Submitted: March 25, 1996; Accepted: April 20, 1996

\begin{abstract}
Following [2], we say a family, $H$, of subsets of a $n$-element set is cancellative if $A \cup B=A \cup C$ implies $B=C$ when $A, B, C \in H$. We show how to construct cancellative families of sets with $c 2^{54797 n}$ elements. This improves the previous best bound $c 2^{.52832 n}$ and falsifies conjectures of Erdös and Katona [3] and Bollobas [1].
\end{abstract}

\section{AMS Subject Classification. 05C65}

We will look at families of subsets of a $n$-set with the property that $A \cup B=A \cup C \Rightarrow$ $B=C$ for any $A, B, C$ in the family. Frankl and Füredi [2] call such families cancellative. We ask how large cancellative families can be. We define $f(n)$ to be the size of the largest possible cancellative family of subsets of a $n$-set and $f(k, n)$ to be the size of the largest possible cancellative family of $k$-subsets of a $n$-set.

Note the condition $A \cup B=A \cup C \Rightarrow B=C$ is the same as the condition $B \triangle C \subseteq$ $A \Rightarrow B=C$ where $\triangle$ denotes the symmetric difference.

Let $F_{1}$ be a family of subsets of a $n_{1}$-set, $S_{1}$. Let $F_{2}$ be a family of subsets of a $n_{2^{-}}$ set, $S_{2}$. We define the product $F_{1} \times F_{2}$ to be the family of subsets of the $\left(n_{1}+n_{2}\right)$-set, $S_{1} \cup S_{2}$, whose members consist of the union of any element of $F_{1}$ with any element of $F_{2}$.

It is easy to see that the product of two cancellative families is also a cancellative family $\left(\left(A_{1}, A_{2}\right) \cup\left(B_{1}, B_{2}\right)=\left(A_{1}, A_{2}\right) \cup\left(C_{1}, C_{2}\right) \Rightarrow\left(A_{1} \cup B_{1}, A_{2} \cup B_{2}\right)=\left(A_{1} \cup C_{1}, A_{2} \cup\right.\right.$ $\left.C_{2}\right) \Rightarrow A_{1} \cup B_{1}=A_{1} \cup C_{1}$ and $A_{2} \cup B_{2}=A_{2} \cup C_{2} \Rightarrow B_{1}=C_{1}$ and $B_{2}=C_{2} \Rightarrow$ $\left.\left(B_{1}, B_{2}\right)=\left(C_{1}, C_{2}\right)\right)$. Hence $f\left(n_{1}+n_{2}\right) \geq f\left(n_{1}\right) f\left(n_{2}\right)$. Similarly $f\left(k_{1}+k_{2}, n_{1}+n_{2}\right) \geq$ $f\left(k_{1}, n_{1}\right) f\left(k_{2}, n_{2}\right)$. 
It is easy to show that $f\left(n_{1}+n_{2}\right) \geq f\left(n_{1}\right) f\left(n_{2}\right)$ implies that $\lim _{n \rightarrow \infty} \frac{1}{n} l g(f(n))$ exists ( $l g$ means $\log$ base 2). Let this limit be $\alpha$. Note that $\alpha \geq \frac{1}{n} l g(f(n))$ for any fixed $n$.

Clearly $f(1, n)=n$ as we may take all the 1-element sets. Let $H_{n}$ be the family of all 1-element sets of a $n$-set. It had been conjectured that the largest cancellative families could be built up by taking products of the families $H_{n}$. For example Bollobas conjectured [1] that

$$
f(k, n)=\prod_{i=1}^{k}[(n+i-1) / k]
$$

which comes from letting $n=n_{1}+\cdots+n_{k}$ where the $n_{i}$ are as nearly equal as possible and considering the family $H_{n_{1}} \times \cdots \times H_{n_{k}}$. When $k=2$ determining $f(2, n)$ is the same as determining how many edges a triangle-free graph can contain. So in this case (1) follows from Turan's theorem. Bollobas [1] proved (1) for $k=3$. Sidorenko [4] proved (1) when $k=4$. Frankl and Füredi [2] proved (1) for $n \leq 2 k$. However, we will show below that (1) is false in general.

Also Erdös and Katona conjectured (see [3]) that (for $n>1$ ) the families achieving $f(n)$ could be built up as products of $H_{3}$ and $H_{2}$ taking as many $H_{3}$ 's as possible. So for example

$$
f(3 m)=3^{m}
$$

This would mean $\alpha=\frac{l g 3}{3}=.52832+$. However, as we will see this conjecture is false as well. In fact we show $\alpha \geq .54797+$.

We now describe the construction which is the main result of this paper. Fix $m \geq 3$. Chose $m-1$ integers $n_{1}, \ldots, n_{m-1}$ from $\{0,1,2\}$ so that $n_{1}+\cdots+n_{m-1} \equiv 0 \bmod 3$. Chose an integer $h$ from $\{1, \ldots, m\}$. Clearly these choices can be made in $m 3^{m-2}$ ways. We now form a cancellative family of subsets of a $3 m$-set containing $m 3^{m-2}$ elements as follows. Identify subsets of a $3 m$-set with 0,1 vectors of length $3 m$ in the usual way. Let the $3 m$ vectors consist of $m$ subvectors of length 3 . Let $v_{0}=(100), v_{1}=(010), v_{2}=(001)$ and $w=(111)$. Form a $3 m$-vector from our choices above as follows. Let the $h$ th 3 subvector be $w$. Let the remaining $m-13$-subvectors be $v_{n_{1}}, \ldots, v_{n_{m-1}}$ in order. Let $F$ be the family consisting of all $3 m$-vectors we can form in this way. Clearly each of the $m 3^{m-2}$ choices gives a different vector so $F$ contains $m 3^{m-2}$ elements. We claim $F$ is a cancellative family. For let $B, C$ be two different vectors in $F$ and look at $B \triangle C$. We claim $B \triangle C$ contains at least two 3 -subvectors with two 1's. There are two cases. If the 3 -subvector $w$ is in different positions in $B$ and $C$ then the 3 -subvectors in $B \triangle C$ in these positions contain two 1's. Alternatively, if the 3 -subvector $w$ is in the same position in $B$ and $C$ then the condition $n_{1}+\cdots+n_{m-1} \equiv 0 \bmod 3$ insures that at least two of the $n_{i}$ differ between $B$ and $C$ (assuming $B$ and $C$ are distinct) and the 3 -subvectors in these positions of $B \triangle C$ contain two 1's. However, this means $B \triangle C \subseteq A \in F$ is impossible (unless $B=C$ ) because all elements of $F$ contain only one 3 -subvector containing two or more 1's.

Hence we have

$$
f(3 m) \geq m 3^{m-2}
$$


THE EleCtronic JoURnal of COMBinatorics 3 (1996), \#R15

$$
f(m+2,3 m) \geq m 3^{m-2} .
$$

Clearly (3) is better than (2) for $m>9$. We also have $\alpha \geq \frac{1}{3 m} \lg \left(m 3^{m-2}\right)$. This is maximized for $m=24$ giving $\alpha \geq .54797+$. So we have counter examples to the Erdös and Katona conjecture.

Furthermore (4) is better than (1) for $m \geq 8$. So the Bollobas conjecture fails for $k \geq 10$.

The idea of the above construction which improves on products of $H_{3}$ can be applied to products of other families as well. For example, we can do better than (1) starting with products of $H_{k}$ for any $k>3$ as well. Or we can start with the families $F$ constructed above. This will allow a very slight improvement in the lower bound found for $\alpha$ above.

The best upper bound known for $\alpha, \alpha<\lg (3 / 2)=.58496+$, is due to Frankl and Füredi [2].

The author thanks Don Coppersmith for bringing this problem to his attention.

\section{REFERENCES}

[1] B. Bollobás, Three-Graphs without two triples whose symmetric difference is contained in a third, Discrete Mathematics 8 (1974), 21-24.

[2] P. Frankl and Z. Füredi, Union-free Hypergraphs and Probability Theory, European Journal of Combinatorics 5 (1984), 127-131.

[3] G.O.H. Katona, Extremal Problems for Hypergraphs, Combinatorics, Mathematical Centre Tracts part 256 (1974), 13-42.

[4] A.F. Sidorenko, The Maximal Number of Edges in a Homogeneous Hypergraph containing no prohibited subgraphs, Mathematical Notes 41 (1987), 247-259 (translation from Mathematicheskie Zametki 41 (1987), 433-455). 